Proceedings

\title{
Chromosome Instability in Asthma an Antioxidant Treatment as a Possible Pharmacological Strategy? ${ }^{\dagger}$
}

\author{
Cláudia Oliveira ${ }^{1, *}$, Maria Nogueira ${ }^{1}$ Telma Barbosa ${ }^{2}$, Carmen Cardoso ${ }^{2}$, João Neves ${ }^{3}$, Solange \\ Costa ${ }^{4,5}$ and Beatriz Porto ${ }^{1}$ \\ 1 Cytogenetics Laboratory, ICBAS-UP, Porto, Portugal; micas22.maria@gmail.com (M.N.); \\ bporto@icbas.up.pt (B.P.) \\ 2 Department of Pediatrics, CMIN-CHUP, Porto, Portugal; claudia.sofia@live.com.pt (T.B.); \\ claudia.sofia.s.oliveira@gmail.com (C.C.) \\ 3 Internal Medicine Department, CHUP, Porto, Portugal; jtcneves@gmail.com \\ 4 Environmental Health Department, INSA, Porto, Portugal; solange.costa@insa.min-saude.pt \\ 5 EPIUnit-ISPUP, Porto, Portugal \\ * Correspondence: csoliveira@icbas.up.pt \\ + Presented at the 1st International e-Conference on Antioxidants in Health and Disease, 01-15 December \\ 2020; Available online: https://cahd2020.sciforum.net/.
}

Published: 30 November 2020

\begin{abstract}
Asthma is a disease with multiple phenotypes and different degrees of severity. Severe inflammation appears when oxidative stress (OS) overwhelms the antioxidant defense. Knowing the role of $\mathrm{OS}$ in the increase of chromosome instability (CI) and, consequently, in cancer predisposition, it is important to carry out studies to establish limits for harmful effects. The aim of this study was to evaluate OS-related CI in asthma patients and the possible protective effect of antioxidants. For that purpose, spontaneous and OS-induced lymphocyte cultures from patients with mild and severe asthma, and from normal controls, were performed. Antioxidant-enriched cultures from severe patients were posteriorly performed. A hundred metaphases per culture were scored in order to estimate the frequency of CI. Our results showed that lymphocytes from severe patients had increased CI, both in spontaneous and OS-induced cultures. However, in lymphocytes from mild patients there were no differences compared to controls. This suggests that in patients subjected to extreme OS conditions, a genotoxic effect may occur at the cellular level. When lymphocytes from these patients were treated with antioxidants, a decrease in CI was observed. Understanding how $\mathrm{CI}$ correlates to asthma patients' clinical situation may be pivotal to the design of future preventive measures.
\end{abstract}

Keywords: asthma; chromosome instability; oxidative stress

\section{Introduction}

Asthma is one of the most common respiratory diseases in the world, with more than 300 million patients diagnosed [1]. It is described as a chronic disease of the respiratory system, with inflammation of the bronchial airways as the most prominent pathological feature [2]. The typical symptoms of asthma are wheezing, chest tightness, shortness of breath, evidence of airway obstruction and airway hyperresponsiveness (AHR). Although originally believed to be a single disease, it is now recognized as a complex of multiple phenotypes, each with different severity and treatment response [3]. 
The 1st International Electronic Conference on Antioxidants in Health and Disease, 1-15 December 2020

Oxidant-antioxidant imbalance plays an important role in the airway inflammation observed in asthmatic patients. Inflammatory cells infiltrating the airways produce several modulators of the inflammatory response, including a range of toxic reactive oxygen species (ROS), which are associated with many of the pathophysiological changes linked with asthma severity, such as increased airway reactivity and secretions, and increased production of chemoattractants [4]. In fact, when ROS overwhelm antioxidant defenses the severe inflammatory state becomes apparent.

Several studies showed that the interaction between ROS and DNA can produce structural alterations including small-scale insertions, DNA base pair deletions, base modifications, aneuploidy, microsatellite instability, translocation of segments and CI [5]. In general, ROS is the major source of DNA damage that attacks phosphate, deoxyribose, and base sites, resulting in strand breakage. Among these, double-strand breaks (DSBs) are one of the most cytotoxic forms of DNA damage, and if they are not properly repaired, they can lead to cell death and genomic rearrangements commonly found in cancer cells [6].

The protective effect of the antioxidant cocktail, $N$-Acetyl-L-cysteine (NAC) and ( \pm )- $\alpha$-Lipoic acid (ALA), against DSBs measured by CI was previously shown in a study involving FA patients, who are characterized by increased levels of OS and CI [7]. Since NAC and ALA, independently, were found to suppress airway inflammation and AHR in animal models of asthma, we hypothesize if the same antioxidant cocktail used in FA patients can have also a protective effect in asthmatic patients with severe inflammation caused by OS [8,9].

The aim of this study was to evaluate OS-related CI in asthma patients and to study the protective effect of in-vitro administration of NAC and ALA.

\section{Experiments}

This study included 19 asthma patients. These individuals were divided in two different groups: a "severe asthma" group $(n=15)$, if they had frequent asthma attacks and needed daily medication, and a "mild asthma" group $(n=4)$ if they were clinically diagnosed with asthma, but without an attack in the last couple of months and without the need of daily medication. As a control group, healthy blood donors $(n=20)$ were recruited.

Initially, three sets of experiments were performed. In the first one, lymphocyte cultures were maintained for $72 \mathrm{~h}$ without any type of treatment, in order to evaluate the spontaneous CI in cells from all groups of individuals. In the other two experiments, $( \pm)-1,2: 3,4$-diepoxibutane (DEB), at the concentrations of $0.05 \mu \mathrm{g} / \mathrm{mL}$ and $0.1 \mu \mathrm{g} / \mathrm{mL}$ respectively, was added $24 \mathrm{~h}$ after culture initiation, in order to evaluate the OS-related induced CI in cells from all groups of individuals.

Afterwards, three new sets of experiments were performed with cells from individuals presenting increased levels of CI. In the first one, lymphocyte cultures were treated with an antioxidant cocktail containing $20 \mu \mathrm{M}$ of ALA (Sigma-Aldrich, St. Louis, MO, USA) and $500 \mu \mathrm{M}$ of NAC (Sigma-Aldrich, St. Louis, MO, USA) $24 \mathrm{~h}$ after culture initiation, in order to evaluate its effect on spontaneous CI. In the other two experiments, lymphocyte cultures were pre-treated with the referred antioxidant cocktail 1 h30 before DEB exposure (at the same conditions described above), in order to evaluate its effect on OS-related induced CI. The selected concentrations of antioxidants were choosen according to previous in vitro studies $[7,10]$.

After 3 days of culture, cells were harvested after $1 \mathrm{~h}$ of incubation with colcemid (Gibco, Invitrogen Corporation, Carlsbad, CA, USA) at a concentration of $4 \mu \mathrm{g} / \mathrm{mL}$, followed by hypotonic treatment with $75 \mathrm{mM} \mathrm{KCl}$ (Sigma-Aldrich, St. Louis, MO, USA) and fixed 3 times in solution of acetic acid (Fisher Chemicals, Thermo Fisher Scientific inc., Waltham, MA, USA) and methanol (Fisher Chemicals, Thermo Fisher Scientific inc., Waltham, MA, USA) in a ratio of 1:3. The resulting suspensions were dropped onto microscope slides and stained for $4 \mathrm{~min}$ in a $4 \%$ Giemsa solution (Merck, Darmstadt, Germany).

Analysis was performed on 100 metaphases from each experiment, by two independent scorers and in a blinded fashion. Each cell was scored for chromosome number and the number and types of 
The 1st International Electronic Conference on Antioxidants in Health and Disease, 1-15 December 2020 structural abnormalities. The parameters analyzed were percentage of aberrant cells and mean number of breaks per cell.

Statistical analysis was performed using the GraphPad Prism 8, version 8.4.3 software. Graphical results are expressed as mean \pm SEM and tabular results are expressed as mean \pm SEM. Statistical comparison between groups was done using a Two-factor mixed-design ANOVA followed by a multicomparison Turkey's test.

\section{Results}

\subsection{Evaluation in Lymphocyte Cultures from Asthma Patients}

\subsubsection{Spontaneous CI}

As shown in Figure 1, lymphocyte cultures from severe asthma patients have a significant increase in the levels of spontaneous CI when compared to controls, either in percentage of aberrant cells $(p<0.0001)$ or mean number of breaks per cell $(p<0.0001)$. Regarding lymphocyte cultures from mild asthma patients, no significant differences were observed in the levels of spontaneous CI when compared to controls, either in percentage of aberrant cells or mean number of breaks per cell.

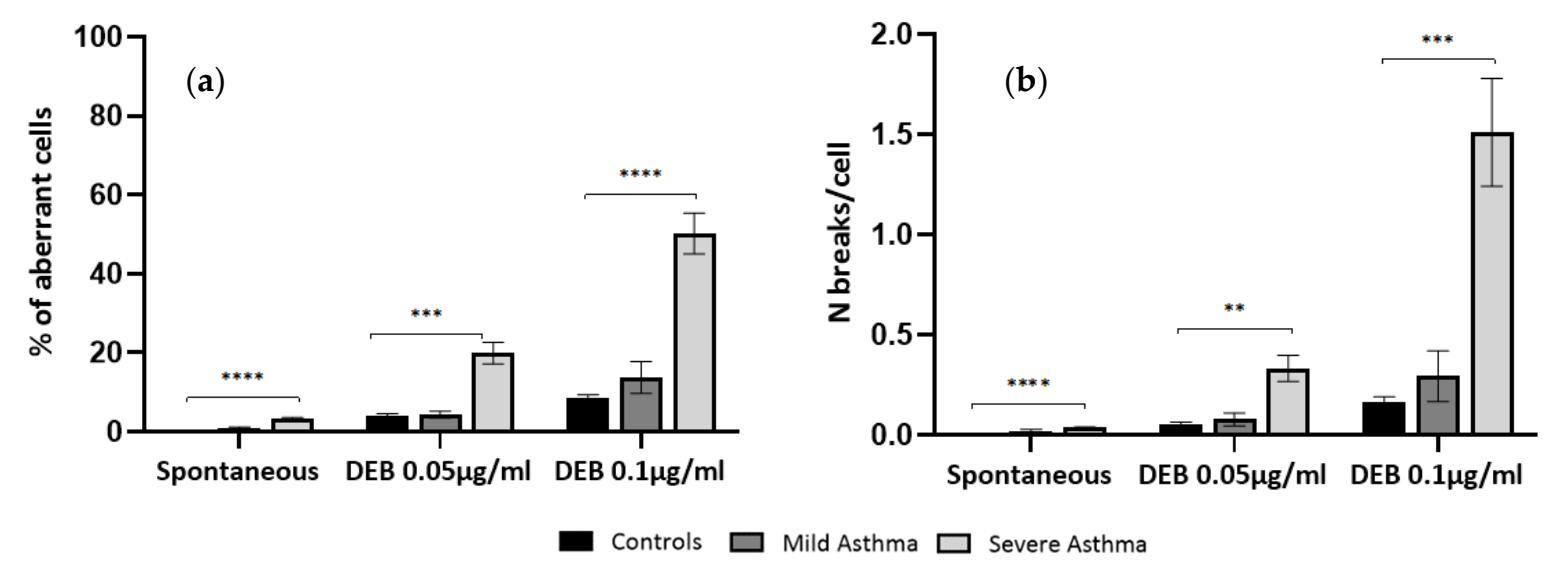

Figure 1. Comparative analysis between the levels of CI in in cultured lymphocytes from controls, mild and severe asthma patients. (a) Percentage of aberrant cells; (b) Mean number of breaks per cell. Data are expressed as means \pm SEM. Differences between groups were evaluated by Two-factor mixed-design ANOVA followed by a multicomparison Turkey's test $(\alpha=0.05):^{* * * *} p<0.0001,{ }^{* * *} p<$ $0.001,{ }^{* *} p<0.01,{ }^{*} p<0.05$.

\subsubsection{OS-Related Induced CI}

As shown in Figure 1, DEB-induced lymphocyte cultures from severe asthma patients have a significant increase in the levels of OS-related CI, when compared to controls, both at $0.05 \mu \mathrm{g} / \mathrm{mL}$ and $0.1 \mu \mathrm{g} / \mathrm{mL}$ DEB concentrations, either in percentage of aberrant cells $(p<0.001$ and $p<0.0001$, respectively) or mean number of breaks per cell ( $p<0.01$ and $p<0.001$, respectively). Figure 2 is an example of the mild DEB-induced CI in a cell from a normal individual, where only a chromatid break is seen, and the increased DEB-induced CI in a cell from a severe asthma patient, with multiple chromosome breaks and rearrangements.

Regarding DEB-induced lymphocyte cultures from mild asthma patients, no significant differences were observed in the levels of CI when compared to controls, either in percentage of aberrant cells or mean number of breaks per cell. 


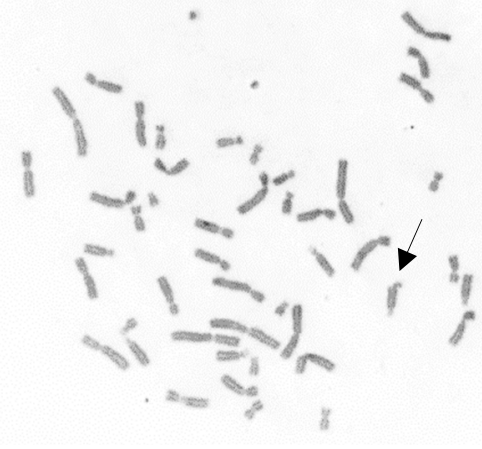

(a)

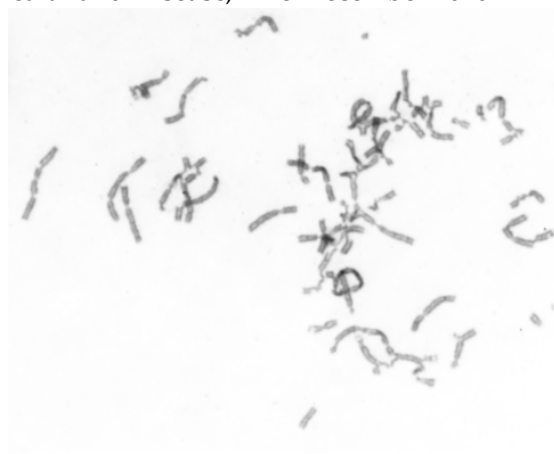

(b)

Figure 2. CI pattern in metaphases from DEB-induced $(0.1 \mu \mathrm{g} / \mathrm{mL})$ lymphocyte cultures (a) Selected metaphase from a control. One chromatid break can be seen; (b) Selected metaphase from a severe asthma patient. High level of chromosome instability can be visualized, with multiple breaks and rearrangements.

\subsection{Antioxidant Effect against CI in Lymphocyte Cultures from Severe Asthma Patients}

\subsubsection{Effect of NAC+ALA on Spontaneous CI}

As shown in Figure 3, lymphocyte cultures from severe asthma patients treated with NAC+ALA have a significant reduction in the percentage of aberrant cells, when compared to cultures of the same group without antioxidant treatment $(p<0.001)$. When comparing the NAC+ALA treated cultures with spontaneous cultures from controls, no differences were observed either in percentage of aberrant cells or number of breaks per cell.

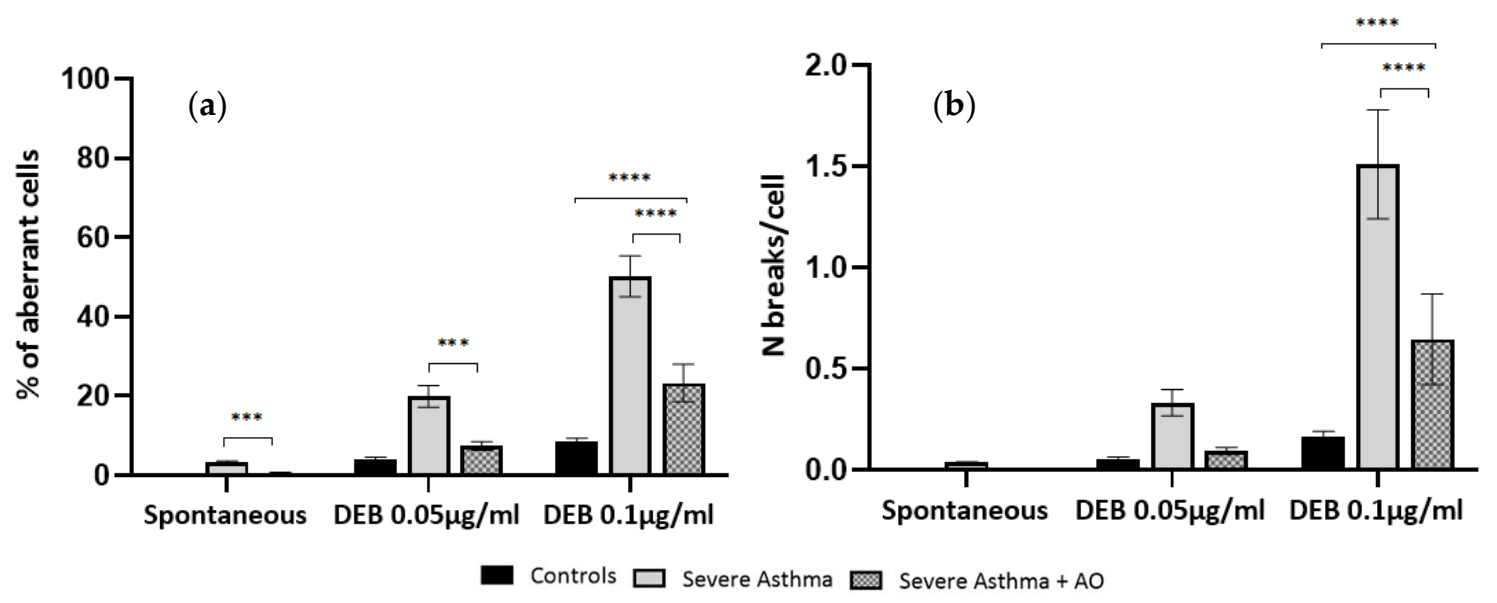

Figure 3. Effect of NAC+ALA in CI in cultured lymphocytes from severe asthma patients. (a) Percentage of aberrant cells; (b) Mean number of breaks per cell. Data are expressed as means \pm SEM. Differences between groups were evaluated by Two-factor mixed-design ANOVA followed by a multicomparison Turkey's test $(\alpha=0.05):{ }^{* * *} p<0.0001,{ }^{* * *} p<0.001,{ }^{* *} p<0.01,{ }^{*} p<0.05$.

\subsubsection{Effect of NAC+ALA on OS-Related Induced CI}

As shown in Figure 3, lymphocyte cultures from severe asthma patients exposed to DEB and pre-treated with NAC+ALA have a significant reduction in the percentage of aberrant cells when compared to those only exposed to DEB, both at $0.05 \mu \mathrm{g} / \mathrm{mL}$ and $0.1 \mu \mathrm{g} / \mathrm{mL}$ DEB concentrations $(p<$ 
The 1st International Electronic Conference on Antioxidants in Health and Disease, 1-15 December 2020

0.001 and $p<0.0001$, respectively). Regarding the number of breaks per cell, there was only a significant difference at the DEB concentration of $0.1 \mu \mathrm{g} / \mathrm{mL}(p<0.0001)$.

When comparing the NAC+ALA pre-treated cultures from severe asthma patients with DEBinduced cultures from controls, only found a significant difference in the number of breaks per cell at the DEB concentration of $0.1 \mu \mathrm{g} / \mathrm{mL}(p<0.0001)$ was found.

\section{Discussion}

The first aim of this study was to evaluate cellular levels of CI in cells from asthma patients. Our results clearly show that cultured lymphocytes from severe asthma patients have increased levels of $\mathrm{CI}$, when compared to controls. Interestingly, no significant differences were found in cells from mild asthma patients. The difference between the two groups may be due to the severity of the disease, and consequently to the inflammatory state present at the time. It is well known that when inflammatory cells infiltrate the airways, several modulators of the inflammatory response are produced, including a range of toxic ROS. Knowing the role of OS in the increase of CI, this pathological feature is in agreement with our results, once in the severe asthma patients' cells a significant increase in the levels of both spontaneous and OS-induced CI were observed. This last result may also indicate that these patients might have a higher sensitivity to the cumulative genotoxic effect of OS, comparatively to normal individuals.

In an attempt to study the possible role of antioxidants in the reduction of levels of CI observed in cells from severe asthma patients, an antioxidant cocktail (NAC+ALA) was added to the lymphocytes cultures. This cocktail was previously proven to be effective in reduction of CI in in vitro studies in patients with increased OS-related CI. Our results clearly show that when exposed to the antioxidant cocktail, cells from severe asthma patients had a significant decrease in the levels of CI, both spontaneous and OS-induced. Further studies are still required in order to understand why these particular antioxidants were effective in the reduction of CI in these cells, namely studies of ROS characterization at cellular level and mitochondrial function.

\section{Conclusions}

In conclusion, the present study provides an important and novel finding that may have a clinical applicability. Understanding how CI due to exposure to OS correlates to asthma patients' clinical phenotype may be pivotal not only to the design of preventive measures, in order to avoid the cumulative OS genotoxic effect, but also to design patient-specific treatments, like the prophylactic use of antioxidants.

Acknowledgments: Authors wish to thank to all volunteers who provided blood samples, through the Service of Hematology from the Hospital Center of Oporto and Institute of Biomedical Science Abel Salazar. They also thank all the asthma patients for participating in this study.

Author Contributions: C.O. designed and performed the experiments, performed research, analyzed data and drafted the manuscript. M.N. performed some of the experiments. T.B., C.C. and J.N. provided blood samples and clinical data from patients, analyzed the experimental data. S.C. coordinated the study and critically revised the manuscript. B.P. coordinated the study, designed experiments, analyzed data, critically revised the manuscript and gave final approval of the version to be published. All authors read and approved the final manuscript.

Conflicts of Interest: The authors declare no conflict of interest. 
The 1st International Electronic Conference on Antioxidants in Health and Disease, 1-15 December 2020

\section{Abbreviations}

The following abbreviations are used in this manuscript:

OS: oxidative stress

CI: chromosome instability

AHR: airway hyperresponsiveness

ROS: reactive oxygen species

DSBs: double-strand breaks

NAC: $N$-acetyl-L-cysteine

ALA: ( \pm$)-\alpha$-Lipoic acid

DEB: ( \pm -1,2:3,4-diepoxibutane

\section{References}

1. Masoli, M.; Fabian, D.; Holt, S.; Beasley, R. The global burden of asthma: Executive summary of the GINA Dissemination Committee report. Allergy 2004, 59, 469-478, doi:10.1111/j.1398-9995.2004.00526.x.

2. Lambrecht, B.N.; Hammad, H. The immunology of asthma. Nat. Immunol. 2015, 16, 45-56, doi:10.1038/ni.3049.

3. White, J.; Paton, J.Y.; Niven, R.; Pinnock, H. Guidelines for the diagnosis and management of asthma: A look at the key differences between BTS/SIGN and NICE. Thorax 2018, 73, 293-297, doi:10.1136/thoraxjnl2017-211189.

4. Rosanna, D.P.; Salvatore, C. Reactive oxygen species, inflammation, and lung diseases. Curr. Pharm. Des. 2012, 18, 3889-3900.

5. Wiseman, H.; Halliwell, B. Damage to DNA by reactive oxygen and nitrogen species: Role in inflammatory disease and progression to cancer. Biochem. J. 1996, 313, 17-29, doi:10.1042/bj3130017.

6. Krejci, L.; Altmannova, V.; Spirek, M.; Zhao, X. Homologous recombination and its regulation. Nucleic Acids Res. 2012, 40, 5795-5818, doi:10.1093/nar/gks270.

7. Ponte, F.; Sousa, R.; Fernandes, A.P.; Gonçalves, C.; Barbot, J.; Carvalho, F.; Porto, B. Improvement of genetic stability in lymphocytes from Fanconi anemia patients through the combined effect of $\alpha$-lipoic acid and N-acetylcysteine. Orphanet J. Rare Dis. 2012, 7, 28, doi:10.1186/1750-1172-7-28.

8. Blesa, S.; Cortijo, J.; Mata, M.; Serrano, A.; Closa, D.; Santangelo, F.; Estrela, J.M.; Suchankova, J.; Morcillo, E.J. Oral $\mathrm{N}$-acetylcysteine attenuates the rat pulmonary inflammatory response to antigen. Eur. Respir. J. 2003, 21, 394-400.

9. Cho, Y.S.; Lee, J.; Lee, T.H.; Lee, E.Y.; Lee, K.U.; Park, J.Y.; Moon, H.B. alpha-Lipoic acid inhibits airway inflammation and hyperresponsiveness in a mouse model of asthma. J. Allergy Clin. Immunol. 2004, 114, 429-435, doi:10.1016/j.jaci.2004.04.004.

10. Ponte, F.; Carvalho, F.; Porto, B. Protective effect of acetyl-l-carnitine and $\alpha$-lipoic acid against the acute toxicity of diepoxybutane to human lymphocytes. Toxicology 2011, 289, 52-58, doi:10.1016/j.tox.2011.07.009.

(C) 2020 by the authors; licensee MDPI, Basel, Switzerland. This article is an open access article distributed under the terms and conditions of the Creative Commons by Attribution (CC-BY) license (http://creativecommons.org/licenses/by/4.0/). 